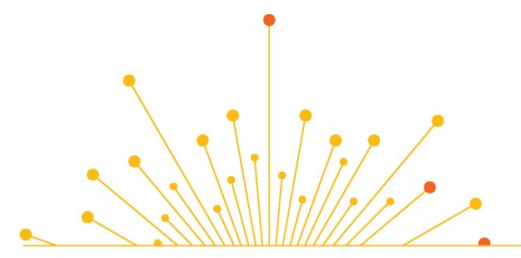

SCIENCE TABLE

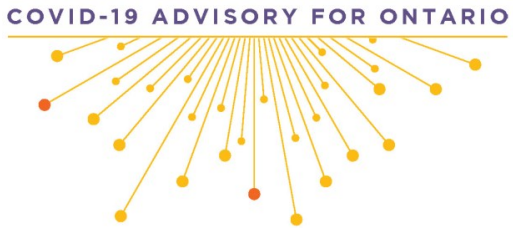

Version 1.0

Published: May 6, 2021

Citation: Morris AM, Jüni $P$, Odutayo A, et al. Remdesivir for hospitalized patients with COVID-19. Science Briefs of the Ontario COVID19 Science Advisory Table. 2021;2(27). https:// doi.org/10.47326/ocsat.2021.02.27.1.0

Author Affiliations: The affiliations of the members of the Ontario COVID-19 Science Advisory Table can be found at https:// covid19-sciencetable.ca/.

Declarations of Interest: The declarations of interest of the members of the Ontario COVID-19 Science Advisory Table can be found at https://covid19-sciencetable.ca/.

About Us: The Ontario COVID-19 Science Advisory Table is a group of scientific experts and health system leaders who evaluate and report on emerging evidence relevant to the COVID-19 pandemic, to inform Ontario's response. Our mandate is to provide weekly summaries of relevant scientific evidence for the COVID-19 Health Coordination Table of the Province of Ontario, integrating information from existing scientific tables, Ontario's universities and agencies, and the best global evidence. The Science Table summarizes its findings for the Health Coordination Table and the public in Science Briefs.

The Drugs \& Biologics Clinical Practice Guidelines Working Group is a group of clinicians and scientists with recognized expertise in drugs, biologics, and clinical care. The Working Group evaluates existing scientific data, disease epidemiology, drug availability, and implementation issues in order to develop Clinical Practice Guidelines for the treatment of COVID-19 using drugs and biologics. The Working Group reports its findings to the public and the Science Table. Its findings are also summarized in Science Briefs.

Correspondence to: Secretariat of the Ontario COVID-19 Science Advisory Table (info@covid19-sciencetable.ca)

Copyright: 2021 Ontario COVID-19 Science Advisory Table. This is an open access document distributed under the terms of the Creative Commons Attribution License,

\section{Remdesivir for Hospitalized Patients with COVID-19}

Andrew M. Morris, Peter Jüni, Ayodele Odutayo, Pavlos Bobos, Nisha Andany, Kali Barrett, Martin Betts, Andrew Healey, Bradley Langford, Antonina Maltsev, Katherine J. Miller, Justin Morgenstern, Laveena Munshi, Fahad Razak, Nathan M. Stall, Menaka Pai on behalf of the Drugs \& Biologics Clinical Practice Guidelines Working Group and the Ontario COVID-19 Science Advisory Table

\section{Key Message}

Remdesivir, a direct-acting antiviral agent, may reduce mortality and progression to mechanical ventilation in moderately ill patients hospitalized with COVID-19 on supplemental low-flow oxygen. The benefits of remdesivir for critically ill patients requiring supplemental oxygen via high-flow nasal cannula or mask, or non-invasive mechanical ventilation, is uncertain. Remdesivir does not benefit and may harm critically ill patients already receiving mechanical ventilation or requiring extracorporeal membrane oxygenation (ECMO), and it does not provide substantial benefit for hospitalized patients who do not require supplemental oxygen. Remdesivir appears to have comparable effects when used for 5 days or 10 days, and does not appear to be associated with significant adverse effects.

Remdesivir is recommended in moderately ill hospitalized patients with COVID-19 requiring supplemental oxygen (Figure 1). Remdesivir may be considered for patients requiring oxygen supplementation via high-flow nasal cannula or mask, or non-invasive mechanical ventilation. It should not be used in critically ill patients on mechanical ventilation or those receiving ECMO. Remdesivir should not be used in patients who do not require supplemental oxygen.

\begin{tabular}{|c|c|c|}
\hline \multicolumn{1}{|c|}{ Severity of Illness } & \multicolumn{1}{|c|}{ Organ Support } & Recommendation \\
\hline Critically III & $\begin{array}{l}\text { Invasive mechanical ventilation or } \\
\text { ECMO }\end{array}$ & Remdesivir is not recommended \\
\hline $\begin{array}{l}\text { High flow oxygen or non-invasive } \\
\text { mechanical ventilation }\end{array}$ & \multicolumn{1}{|c|}{ Remdesivir may be considered } \\
\hline Moderately III & Low flow oxygen & Remdesivir is recommended \\
\hline Mildly III & None & Remdesivir is not recommended \\
\hline
\end{tabular}

Figure 1. Summary of Recommendations for Remdesivir by Severity of COVID-19 IIIness

Please see the section below entitled "Methods Used for this Scientific Brief" for a description of COVID-19 illness severity criteria.

\section{Lay Summary}

\section{Remdesivir Might Save Lives in Hospitalized COVID-19 Patients, with Moderate Illness}

Patients with COVID-19 are ill because of a combination of viral infection and the 
which permits unrestricted use, distribution, and reproduction in any medium, provided that the original work is properly cited.

The views and findings expressed in this Science Brief are those of the authors and do not necessarily reflect the views of all of the members of the Ontario COVID-19 Science Advisory Table, its Working Groups, and its partners. inflammation that their body mounts to respond to that infection. Inflammation can compromise their breathing and damage their lungs, heart, kidney, and other vital organs.

Remdesivir is an antiviral medication approved by Health Canada for patients with severe symptoms of COVID-19. It is approved for adults and children aged 12 years and older who weigh at least $40 \mathrm{~kg}$. Remdesivir might improve survival and might prevent hospitalized patients with moderate severity disease from needing mechanical ventilation (a breathing tube). As a COVID-19 treatment, it should only be considered in hospitalized patients on supplemental oxygen whom doctors determine to be "moderately" ill with COVID-19. It is not appropriate for patients who are either mildly ill with COVID-19 or critically ill with COVID-19 and requiring invasive ventilation.

Importantly, most remdesivir studies were performed before corticosteroids, such as dexamethasone, were widely used to treat COVID-19 patients. Since corticosteroids are effective treatments when prescribed for COVID-19, it is unclear from the remdesivir trial data we reviewed whether remdesivir provides additional benefit.

\section{How Remdesivir Works}

Remdesivir is an antiviral drug that is given intravenously. It works by blocking certain viruses from reproducing, including SARS-CoV-2, the virus that causes COVID-19.

\section{How We Came to Our Recommendations}

To understand remdesivir's effect on patients hospitalized with COVID-19, we reviewed four randomized controlled trials (RCTs) from around the world that compared remdesivir to placebo or to routine care alone. Two of these trials were very large. The results of the trials varied, but when taken together, they showed that remdesivir might decrease death and the need for mechanical ventilation and speeds up recovery when given to moderately ill hospitalized patients with COVID19 on supplemental oxygen.

COVID-19 patients taking remdesivir in these studies very rarely had side-effects; in fact, they were less likely to suffer adverse events than patients in the placebo or routine care groups.

\section{Remdesivir Availability in Ontario}

There is a limited supply of remdesivir in Ontario. For this reason, doctors should be careful about using the drug only when it is needed.

\section{Our Recommendation for Using Remdesivir as a Treatment for COVID-19}

We recommend that patients hospitalized with COVID-19 should receive remdesivir if they have moderate illness requiring supplemental oxygen.

Remdesivir may be considered for patients who need more oxygen support than supplemental oxygenation by nasal prongs, but do not require mechanical ventilation. In these patients, it is uncertain whether remdesivir is helpful.

At this time, we do not recommend remdesivir for patients who are mildly ill with COVID-19.

We also do not recommend remdesivir for patients who are critically ill, requiring mechanical ventilation or ECMO. 


\section{Summary}

\section{Background}

Remdesivir is an antiviral drug that has been shown to be effective against the SARSCoV-2 virus in animal models and laboratory studies. ${ }^{1,2}$ There are currently four RCTs comparing remdesivir to placebo or usual care, ${ }^{3-6}$ and another RCT that compared two different durations of remdesivir without a control group. ${ }^{7}$

In July 2020, Health Canada approved the use of remdesivir for the treatment of hospitalized adults and children (aged 12 years and over with body weights of at least $40 \mathrm{~kg}$ ) with COVID-19 pneumonia requiring supplemental oxygen.

\section{Questions}

Does remdesivir improve patient outcomes such as mortality, need for mechanical ventilation, requirement for organ support, intensive care unit (ICU) length of stay, and hospital length of stay for patients hospitalized with COVID-19?

Which patients hospitalized with COVID-19 will receive the most benefit from remdesivir?

What is the recommended duration of therapy for remdesivir?

\section{Findings}

As of March 1, 2021, four RCTs have been published in peer-reviewed journals, studying the effect of remdesivir compared with placebo or usual care in a total of 7,334 patients hospitalized with COVID-19. Disease severity for the patients with COVID-19 enrolled in these trials ranged from mild (i.e., not requiring supplemental oxygen) to critically ill. A fifth trial compared 5 days of remdesivir therapy to 10 days in patients hospitalized with COVID-19 requiring supplemental oxygen or ventilatory support at randomization, but who were not mechanically ventilated or on ECMO.

It is important to note that all of these trials were completed before the emergence of strong evidence in support of corticosteroids and IL-6 inhibitors like tocilizumab for the treatment of COVID-19.

A meta-analysis of RCTs of the effect of remdesivir on all-cause mortality at 14-28 days in hospitalized patients with COVID-19 irrespective of their severity of illness was inconclusive (pooled risk ratio (RR) 0.93 , 95\% confidence interval ( $\mathrm{Cl}$ ) 0.82 to 1.06). However, for those on supplemental oxygen and not ventilated at baseline, the pooled $\mathrm{RR}$ is $0.82(95 \% \mathrm{Cl} 0.69$ to 0.96$)$, corresponding to an absolute risk reduction of $2.3 \%$ and a number-needed-to-treat to save one life of 44 . For hospitalized patients on no supplemental oxygen, the pooled RR was $0.85(95 \% \mathrm{Cl}$ 0.42 to 1.72 ), similar to patients on supplemental oxygen, but with an absolute risk reduction of $0.3 \%$, and a number-needed-to-treat to save one life of 334 . The RR for critically ill patients on mechanical ventilation or high-flow oxygen was $1.23(95 \% \mathrm{Cl}$ 0.99 to 1.53). The difference in treatment effects in critically ill patients on mechanical ventilation or high-flow oxygen as compared with remaining patients was statistically significant ( $p$-value for interaction $=0.004)$.

Remdesivir reduced the composite of mechanical ventilation or death at day 28, with a RR of 0.71 (95\% Cl 0.57 to 0.88$)$.

In the ACTT-1 trial, the median time to recovery was 2 days shorter for those on supplemental oxygen via nasal prongs at baseline with remdesivir as compared with placebo ( 7 days versus 9 days; RR $1.45,95 \% \mathrm{Cl} 1.18$ to 1.79). In two smaller RCTs, remdesivir shortened the time to clinical improvement, with a pooled hazard ratio 
of 1.17 ( $95 \% \mathrm{Cl} 1.02$ to 1.34$)$.

Remdesivir versus placebo may reduce hospital length of stay, with a mean reduction of 5 days ( $95 \% \mathrm{Cl} 2.3$ to 7.7 days). Because the SOLIDARITY trial required patients to remain in hospital to complete a full course of treatment, the study design biased the trial towards no effect on hospital length of stay.

Based on all available data, serious adverse events were significantly lower in patients receiving remdesivir as compared with placebo or usual care (RR $0.75,95 \%$ $\mathrm{Cl} 0.63$ to 0.89 ). The most common reported serious adverse events were respiratory failure, and the most common non-serious adverse events reported were decreased renal function, anemia, lymphopenia, respiratory failure, fever, and hyperglycemia.

There are two published RCTs that address the duration of therapy with remdesivir. One trial compared remdesivir 5 days versus 10 days in 397 patients with mild to moderate illness, whereas the other trial compared remdesivir 5 days with 10 days or usual care in a 1:1:1 ratio in 596 patients (402 in the two remdesivir arms) with moderate to critical illness. The different dosing regimens had no effect on 28-day mortality ( $R R=.87,95 \% \mathrm{Cl} 0.53$ to 1.40 ) or on the 28-day incidence of clinical improvement (RR $1.06,95 \% \mathrm{Cl} 0.87$ to 1.30 ). There were fewer adverse events with the 5 -day regimen ( $\mathrm{RR} 0.65,95 \% \mathrm{Cl} 0.48$ to 0.89 ).

\section{Practical Considerations}

There is a limited supply of remdesivir in Ontario. For this reason, clinicians should be selective about using the drug only when it is needed. In Ontario, remdesivir is distributed to hospitals through the Canadian Pharmaceutical Distribution Network (CPDN). In order to maximize equity across the province and maintain a stable supply, clinicians are encouraged to follow evidence-based principles in prescribing remdesivir. Some strategies for tocilizumab are also applicable to remdesivir. ${ }^{8}$

\section{Recommendations}

Please see the section below entitled "Methods Used for this Scientific Brief" for a description of COVID-19 illness severity criteria.

\section{Critically III Patients (requiring high-flow oxygen, ventilation or ECMO):}

Remdesivir is not recommended for critically ill patients with COVID-19 receiving mechanical ventilation or ECMO.

In patients requiring high-flow oxygen (i.e., oxygen by mask, oxygen by high-flow nasal cannula, or non-invasive mechanical ventilation), remdesivir $200 \mathrm{mg}$ day 1 then $100 \mathrm{mg}$ daily for 4 days may be considered for suspected or confirmed COVID-19.

\section{Moderately III Patients (requiring low-flow supplemental oxygen):}

Remdesivir $200 \mathrm{mg}$ day 1 then $100 \mathrm{mg}$ daily for 4 days is recommended for patients who are moderately ill with suspected or confirmed COVID-19.

Mildly III Patients (not requiring new or additional oxygen):

Remdesivir is not recommended for patients who are mildly ill with suspected or confirmed COVID-19.

\section{Background}

Infection with SARS-CoV-2, the virus causing COVID-19, begins with the virus binding to the human ACE-2 receptor, followed by a period of viral replication and release of 
the virus in the lungs. Initial symptoms, which include fever, muscle aches, headache, and respiratory symptoms, are usually mild or absent, and start developing approximately 1 to 5 days after initial infection. ${ }^{9}$

Viral replication appears to peak 5 to 9 days after initial infection, before the host immune response - characterized by SARS-CoV-2-specific T-cell mediated immunity - attempts to contain it. It is believed that SARS-CoV-2 can cause a variety of symptoms and signs outside of the lungs because ACE- 2 receptors are found in various sites throughout the body.

This vigorous immune response to infection can result in organ damage, with diffuse inflammation of lung alveoli, neutrophilic infiltrates, and microvascular thrombosis. This aberrant hyperactivation of the immune system is an important therapeutic target in COVID-19, and corticosteroids (such as dexamethasone) and interleukin-6 inhibitors (such as tocilizumab) have already demonstrated convincing beneficial effects in COVID-19. ${ }^{10-12}$

Coronaviruses, like SARS-CoV-2, use an RNA-dependent RNA polymerase (RdRp), to carry out replication and transcription of their RNA genome. ${ }^{10}$ Remdesivir is an antiviral drug effective against the SARS-COV-2 virus in animal models and laboratory studies. ${ }^{1,2}$ It is a nucleoside analog prodrug that inhibits coronavirus RdRp when it is metabolized to remdesivir triphosphate and is incorporated into the growing viral RNA product, whose growth is then stalled. ${ }^{13}$

There are currently four RCTs comparing remdesivir to placebo or usual care, ${ }^{3-6}$ and another RCT that compared two different durations of remdesivir without a control group. $^{7}$

In July 2020, Health Canada approved, with conditions, the use of remdesivir for the treatment of hospitalized adults and children (aged 12 years and over with body weights of at least $40 \mathrm{~kg}$ ) with COVID-19-associated pneumonia requiring supplemental oxygen. The Government of Canada procured 150,000 vials of remdesivir in September 2020, which corresponds to 25,000 treatment courses of 5 days duration. Because remdesivir's only indication is COVID-19 infection and is manufactured by a single producer, the Canadian supply of remdesivir is limited.

\section{Questions}

Does remdesivir improve patient outcomes such as mortality, need for mechanical ventilation, requirement for organ support, ICU length of stay, and hospital length of stay for patients hospitalized with COVID-19?

Which patients hospitalized with COVID-19 will receive the most benefit from remdesivir?

What is the recommended duration of therapy for remdesivir?

\section{Findings}

As of April 14, 2021, four RCTs have been released, either as preprints or in peerreviewed journals, studying the effect of remdesivir on a total of 7,334 patients hospitalized with COVID-19. ${ }^{3-6}$ There is heterogeneity in COVID-19 disease severity in enrolled patients, ranging from mildly ill patients not requiring supplemental oxygen, to critically ill patients receiving invasive mechanical ventilation. A fifth trial compared 5 days of remdesivir therapy with 10 days in patients hospitalized with COVID-19 requiring supplemental oxygen or ventilatory support at randomization who were not mechanically ventilated or on ECMO. ${ }^{7}$

It is important to note that all of these trials were completed before the emergence 
of strong evidence in support of corticosteroids and IL-6 inhibitors in COVID-19. ${ }^{10-12}$

Of the four RCTs, two were placebo-controlled, ${ }^{5,6}$ and two randomized patients to remdesivir or usual care. ${ }^{3,4}$ One of these trials compared remdesivir 5 days versus remdesivir 10 days, but also compared each against placebo. A fifth trial compared remdesivir 5 days versus 10 days. ${ }^{7}$

Multiple meta-analyses for remdesivir including these five trials are currently available in peer-reviewed journals. ${ }^{4,14,15}$

\section{Outcome: Overall Mortality}

Figure 2 presents a meta-analysis of all released RCTs in hospitalized patients with COVID-19, irrespective of their severity of illness, comparing remdesivir with placebo or usual care on all-cause mortality at 14-28 days from initiation of therapy. Results are inconclusive, with a pooled RR of 0.93 (95\% Cl 0.82 to 1.06).

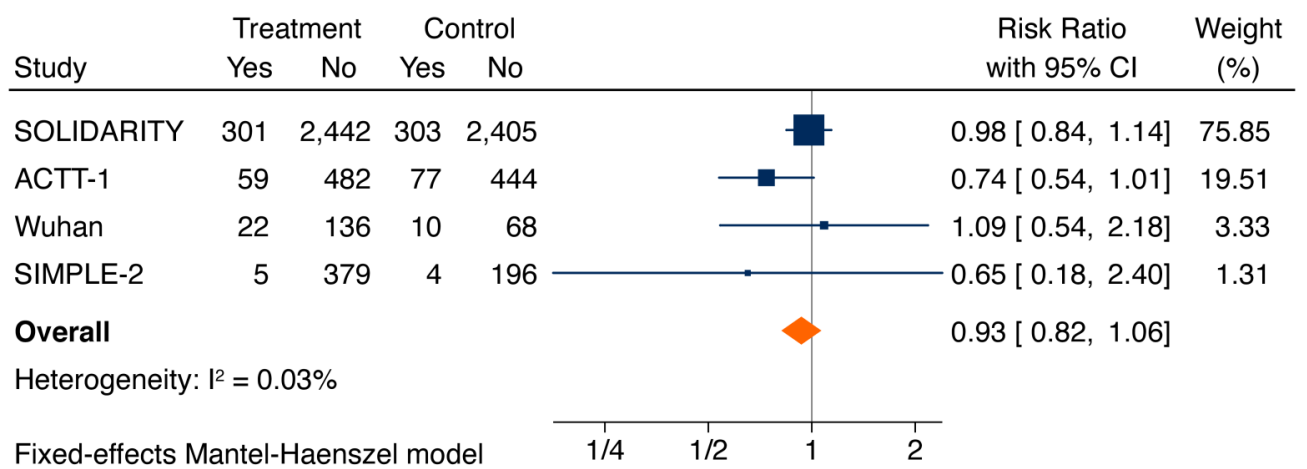

Figure 2. Meta-Analysis of the Effect of Remdesivir Versus Control on All-Cause Mortality in RCTs of Patients Hospitalized With COVID-19

Forest plot presenting the RRs of all-cause mortality comparing remdesivir versus control from RCTs in patients hospitalized with COVID-19. Each square presents the results of an individual RCT with the size of the square being proportional to the weights used in the meta-analysis and the horizontal lines indicating the 95\% Cls. The solid vertical line represents no effect on mortality and the solid diamond indicates the overall summary estimate.

Figure 3 shows meta-analyses of the impact of remdesivir on mortality among patients hospitalized with COVID-19 and stratified by the extent of respiratory support. For hospitalized COVID-19 patients on supplemental oxygen and not ventilated at baseline, the pooled $\mathrm{RR}$ was 0.82 ( $95 \% \mathrm{Cl} 0.69$ to 0.96 ), with a corresponding absolute risk reduction of $2.3 \%$ and a number-needed-to-treat to save one life of 44 . For hospitalized patients on no supplemental oxygen the pooled RR was 0.85 (95\% Cl 0.42 to 1.72), similar to patients on supplemental oxygen, but with an absolute risk reduction of $0.30 \%$ and a number-needed-to-treat to save one life of 334. In contrast, the pooled RR for patients on mechanical ventilation was 1.23 (95\% $\mathrm{Cl} 0.99$ to 1.53 ). The difference in treatment effects in critically ill patients on mechanical ventilation or high-flow oxygen as compared with remaining patients was statistically significant ( $p$-value for interaction $=0.004)$. 


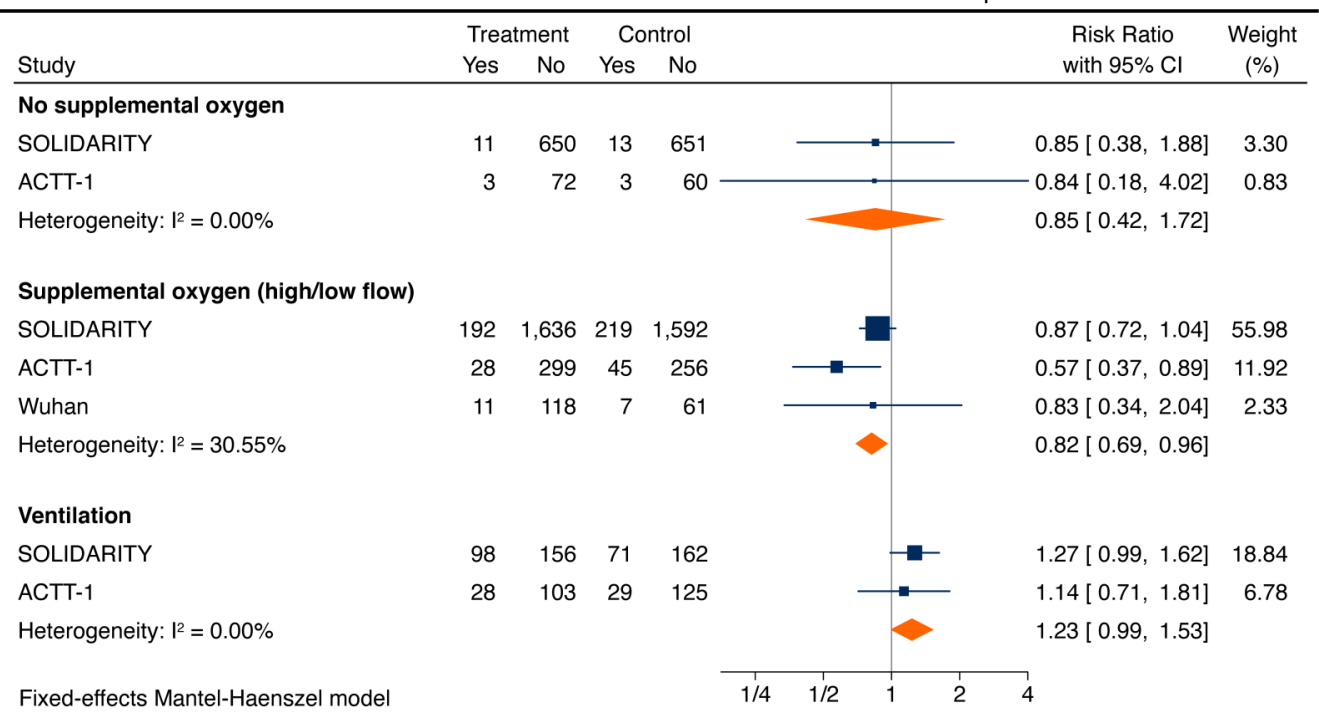

Figure 3. Meta-Analyses of the Effect of Remdesivir Versus Control on All-Cause Mortality by Extent of Respiratory Support

Forest plot presenting the RRs of all-cause mortality by extent of respiratory support at baseline: patients on no supplemental oxygen at baseline, patients on supplemental oxygen but not mechanically ventilated at baseline, and patients ventilated at baseline. Each square presents the results of an individual RCT with the size of the square being proportional to the weights used in the meta-analysis and the horizontal lines indicating the 95\% Cls. The solid vertical line represents no effect on mortality and the diamonds indicate the overall summary estimate.

SOLIDARITY, an open-label trial, looked at 5,451 patients, the majority of whom were on supplemental oxygen at study entry, and found no difference in overall 28day mortality ( $12.5 \%$ remdesivir versus $12.7 \%$ usual care; RR $0.98,95 \% \mathrm{Cl} 0.84$ to 1.14). There were no significant differences in treatment effects between patients on mechanical ventilation at randomization and remaining patients, but the estimated rate ratio for death was 1.20 in patients on mechanical ventilation $(95 \% \mathrm{Cl}$ 0.80 to 1.80 ) and 0.86 in remaining patients ( $95 \% \mathrm{Cl} 0.67$ to 1.11$)^{4}$

ACTT-1, a placebo-controlled trial, looked at 1,062 patients, the majority of whom were on supplemental oxygen at study entry, and found no statistically significant difference in overall 28 -day mortality (11.4\% remdesivir versus $15.2 \%$ placebo) with a hazard ratio of $0.73(95 \% \mathrm{Cl} 0.52$ to 1.03$)$. The estimated rate ratio for death was 0.30 ( $95 \% \mathrm{Cl} 0.11$ to 0.81 ) for patients on low-flow oxygen. ${ }^{6}$

\section{Outcome: Mechanical Ventilation or Death}

Figure 4 shows a meta-analysis of the composite of mechanical ventilation or death. Based on all available data, remdesivir reduced the risk of mechanical ventilation or death at 28 days (RR $0.72,95 \% \mathrm{Cl} 0.57$ to 0.90 ). ${ }^{3,5,6}$

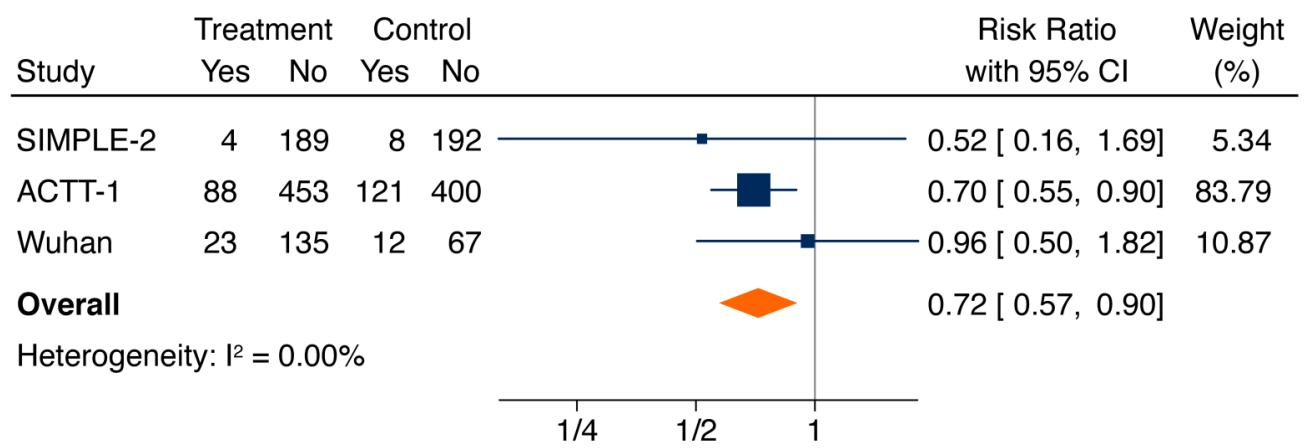

Fixed-effects Mantel-Haenszel model

Figure 4. Meta-Analysis of the RR of Mechanical Ventilation or Death at Day 28 With Remdesivir Versus Control in RCTs of Patients Hospitalized With COVID-19

Forest plot presenting the RRs of the composite of mechanical ventilation or death comparing remdesivir versus 
control from RCTs in patients hospitalized with COVID-19. Each square presents the results of an individual RCT with the size of the square being proportional to the weights used in the meta-analysis and the horizontal lines indicating the $95 \% \mathrm{Cls}$. The solid vertical line represents no difference in the composite of mechanical ventilation or death and the solid diamond indicates the overall summary estimate. Note that the analysis of the SIMPLE-2 trial and the trial from Wuhan includes only hospitalized patients with COVID-19 who were not on mechanical ventilation at randomization. Conversely, the analysis of ACTT-1 also includes hospitalized patients who were on mechanical ventilation at randomization. Therefore, some patients on mechanical ventilation at randomization in ACTT-1 could be included as patients on mechanical ventilation at 28 days.

\section{ICU Length of Stay}

Published data precluded pooling of trials for ICU length.

\section{Other Outcomes: Need for Organ Support, Hospital Length of Stay, Recovery}

In the three RCTs where it was studied, remdesivir reduced the need for ventilation or ECMO at follow-up, with a pooled RR of 0.71 (95\% Cl 0.56 to 0.90$).{ }^{15}$

In ACTT-1, the median time to recovery was 2 days shorter for those on supplemental oxygen via nasal prongs at baseline given remdesivir compared with placebo ( 7 days versus 9 days; RR 1.45 (95\% Cl 1.18 to 1.79$)){ }^{6}$ In the two smaller RCTs by Wang et al. and Spinner et al., remdesivir shortened the time to clinical improvement, with a pooled hazard ratio of 1.17 (95\% Cl 1.02 to 1.34 ). ${ }^{3,5,16}$

Remdesivir versus placebo may reduce hospital length of stay, with a mean reduction of 5 days ( $95 \% \mathrm{Cl} 2.3$ to 7.7 days). ${ }^{15}$ Because the SOLIDARITY trial required patients to remain in hospital to complete a full treatment course, the study design biased the trial towards no effect on hospital length of stay. ${ }^{4}$ Remdesivir resulted in a higher proportion of patients recovered compared to control, with RR $1.09(95 \% \mathrm{Cl}$ 1.03 to 1.15$)^{3,5,6,15}$

\section{Serious Adverse Effects}

Figure 5 shows a meta-analysis of the serious adverse events. Based on all available data, serious adverse events were significantly lower in patients receiving remdesivir, as compared with placebo or usual care (RR of $0.75,95 \% \mathrm{Cl} 0.63$ to 0.89). ${ }^{3,5,6}$ The most common serious adverse events were respiratory failure, and the most common non-serious adverse effects reported were decreased renal function, anemia, lymphopenia, respiratory failure, fever, and hyperglycemia.

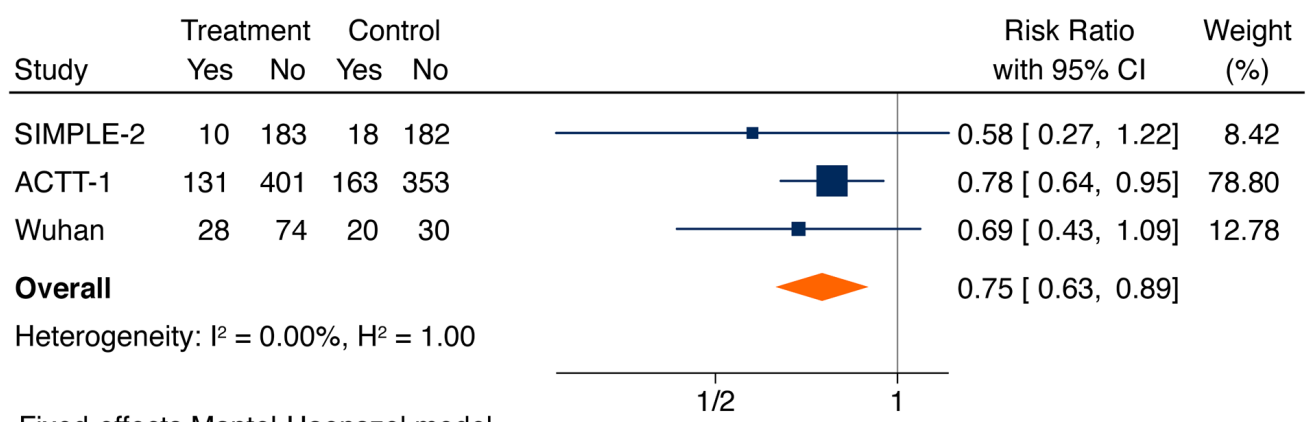

Fixed-effects Mantel-Haenszel model

Figure 5. Meta-Analysis of Serious Adverse Events of Remdesivir Versus Control in RCTs of Patients Hospitalized With COVID-19

Forest plot presenting the RRs of serious adverse events comparing remdesivir versus control from RCTs in patients hospitalized with moderate to severe COVID-19 illness. Each square presents the results of an individual RCT with the size of the square being proportional to the weights used in the meta-analysis and the horizontal lines indicating the 95\% Cls. The solid vertical line represents no effect on serious adverse events and the diamond indicates the overall summary estimate.

\section{Recommended Duration of Therapy}

There are two published RCTs that address duration of therapy with remdesivir. ${ }^{3,7}$ One trial compared remdesivir 5 days versus 10 days in 397 patients with mild to 
moderate illness, ${ }^{7}$ whereas the other trial compared remdesivir 5 days versus 10 days or usual care in a 1:1:1 ratio in 596 patients (402 in the two remdesivir arms) with moderate to critical illness. ${ }^{7}$ There was no significant difference between the two dosing regimens in 28-day mortality (RR $0.87,95 \% \mathrm{Cl} 0.53$ to 1.40) nor in 28-day incidence of clinical improvement (RR $1.06,95 \% \mathrm{Cl} 0.87$ to 1.30). Adverse events were fewer with the 5-day regimen ( $\mathrm{RR} 0.65,95 \% \mathrm{Cl} 0.48$ to 0.89 ).

\section{Interpretation}

The effect of remdesivir on clinical outcomes varies by the severity of COVID-19 illness and extent of respiratory support. In patients hospitalized with moderate COVID-19 illness requiring supplemental oxygen, remdesivir reduces mortality and the composite of mechanical ventilation or death. In these patients, remdesivir also reduces time to clinical improvement and recovery, and length of stay, which has implications for hospital and ICU capacity. Remdesivir does not benefit patients on mechanical ventilation; in these patients the $95 \% \mathrm{Cl}$ of the RR of death is compatible with clinically relevant harm. In patients with mild illness who do not require supplemental oxygen, the risk of death appears too low and the number-needed-totreat to prevent an event too high to justify the use of remdesivir.

The evidence therefore suggests that there is little benefit from remdesivir for patients who are mildly ill and do not require supplemental oxygen and for those who are critically ill requiring mechanical ventilation or ECMO. Patients whose severity is somewhere in between benefit from remdesivir. The severity where benefit is seen likely starts when supplemental oxygen is needed and ends sometime before patients require mechanical ventilation.

With only four RCTs evaluating remdesivir, interpretation of its benefit rests largely on the two largest trials: ACTT-1 and SOLIDARITY. ${ }^{3,4}$ Neither trial was done in the "corticosteroid era" of COVID-19, and thus the additive benefit of remdesivir in patients treated with corticosteroids is unknown. The NIH-sponsored ACTT-1 trial, a placebo-controlled trial, enrolled 541 patients to remdesivir and was underpowered to evaluate a mortality difference, whereas the WHO-led SOLIDARITY trial was an open-label trial with 2,750 randomized to remdesivir.

International recommendations for the use of remdesivir vary. The National Institute of Health recommends the use of remdesivir or dexamethasone in hospitalized patients requiring supplemental oxygen (moderate ("B") strength recommendation), and also recommends it as an option for hospitalized patients receiving oxygen via high-flow device or non-invasive ventilation. ${ }^{17}$ The World Health Organization's (WHO's) guidance, on the other hand, recommends against the use of remdesivir and rates the evidence quality on mortality, mechanical ventilation, time to clinical improvement, and hospital length of stay as low. ${ }^{18}$

There are, at present, relatively few practical considerations surrounding the use of remdesivir in patients hospitalized with COVID-19. Reported side effects in the clinical trials appear limited, and more consistent with progression of disease.

There is a limited supply of remdesivir in Ontario, but there are no projected shortages of the drug. In order to maximize equity across the province, and maintain a stable supply, clinicians are encouraged to follow evidence-based principles in prescribing remdesivir. Some strategies for managing tocilizumab supply during shortage situations may be applicable to remdesivir. ${ }^{8}$ 


\section{Recommendations}

Please see the section below entitled "Methods Used for this Scientific Brief" for a description of COVID-19 illness severity criteria.

\section{Critically III Patients (on high-flow oxygen, mechanical ventilation or ECMO):}

Remdesivir is not recommended for critically ill patients with COVID-19 receiving mechanical ventilation.

In critically ill patients requiring high-flow oxygen (i.e., oxygen by mask, oxygen by high-flow nasal cannula, or non-invasive mechanical ventilation), remdesivir $200 \mathrm{mg}$ day 1 then $100 \mathrm{mg}$ daily for 4 days may be considered for suspected or confirmed COVID-19.

\section{Moderately III Patients (requiring low-flow oxygen):}

Remdesivir $200 \mathrm{mg}$ day 1 then $100 \mathrm{mg}$ daily for 4 days is recommended for patients who are moderately ill with suspected or confirmed COVID-19.

\section{Mildly III Patients (not requiring supplemental oxygen):}

Remdesivir is not recommended for patients who are mildly ill with suspected or confirmed COVID-19.

\section{Methods Used for This Science Brief}

We searched PubMed, Google Scholar, the Joanna Briggs Institute's COVID-19 Special Collection, LitCovid in PubMed, the Oxford COVID-19 Evidence Service, the World Health Organization's Global Literature on Coronavirus Disease, and other COVID-19 specific resources listed by the Guidelines International Network and the McMaster Health Forum. In addition, we retrieved reports citing relevant articles through Google Scholar and reviewed references from identified articles for additional studies. The search was last updated on February 21, 2021.

We conducted our meta-analyses using STATA Release 16.0 software (StataCorp LLC, College Station, TX) using a Mantel-Haenszel fixed-effects model.

For therapeutic recommendations, we used the following definitions for severity:

\section{Critically III}

Patients requiring ventilatory and/or circulatory support, including high-flow nasal oxygen, non-invasive ventilation, invasive mechanical ventilation, or ECMO. These patients are usually managed in an intensive care setting.

\section{Moderately III}

Patients newly requiring low-flow supplemental oxygen. These patients are usually managed in hospital wards.

\section{Mildly III}

Patients who do not require new or additional supplemental oxygen from their baseline status, intravenous fluids, or other physiological support. These patients are usually managed in an ambulatory/outpatient setting.

\section{Author Contributions}

AMM and PJ wrote the first draft of the Science Brief. PB and AO performed the meta-analyses. All authors contributed to the conception of the Science Brief, 
revised it critically for important intellectual content, and approved the final version.

The authors would like to thank all members of the Drugs and Biologics Clinical Practice Guideline Working Group for their contribution to this Science Brief and the Guidelines currently available at https://www.antimicrobialstewardship.com/ and https://covid19-sciencetable.ca/about/

The authors would like to acknowledge the important contributions of the Ontario COVID-19 Antimicrobial and Immunomodulatory Clinical Practice Guidelines Committee throughout the COVID-19 pandemic. Those members include: Karim Ali, MD, Niagara Health System; Amir Amiri, Patient Partner; Nisha Andany, MD MPH, Sunnybrook Health Sciences Centre; Sally Bean, Ethicist, Sunnybrook Health Sciences Centre; Zain Chagla, MD, Hamilton Health Sciences; Bill Ciccotelli, MD, Grand River Hospital ; Pavani Das, MD, North York General Hospital; Linda Dresser, PharmD, University Health Network; Sameer Elsayed, MD, LHSC Victoria Hospital; Wayne Gold, MD, University Health Network; Kevin Gough, MD, St. Michael's Hospital, Unity Health Toronto; Chris Graham, MD, Trillium Health Partners; Rebecca Greenberg, Ethicist, Sinai Health; Shahid Husain, MD MS, University Health Network; Neal Irfan, PharmD BScPhm, Hamilton Health Sciences; Susan John, MD, Scarborough Health Network; Rupert Kaul, MD PhD, University Health Network; Elizabeth Leung, PharmD MSCl, St. Michael's Hospital, Unity Health Toronto; Brian Minnema, MD, St. Joseph's Hospital, Unity Health Toronto; Jeya Nadarajah, MD, MSc, Markham Stouffville Hospital; Caroline Nott, MD, The Ottawa Hospital; Lesley Palmay, BSc BScPhm MSc, Sunnybrook Health Sciences Centre; Alexandra Persichino, BScPhm, Thunder Bay Regional Health Sciences Center; Sumit Raybardhan, MPH, North York General Hospital; Kathryn Timberlake, PharmD, Hospital for Sick Children; Liliana Volnikova, Patient Partner; Anupma Wadhwa, MD MEd, Hospital for Sick Children; Evan Wilson, MD MSc, Kingston Health Sciences Centre; Peter Wu, MD MSc, University Health Network; Ivan Ying, MD, Mackenzie Health.

\section{References}

1. Williamson BN, Feldmann F, Schwarz B, et al. Clinical benefit of remdesivir in rhesus macaques infected with SARS-CoV-2. Nature. 2020;585(7824). https:// doi.org/10.1038/s41586-020-2423-5

2. Wang $M$, Cao $R$, Zhang $L$, et al. Remdesivir and chloroquine effectively inhibit the recently emerged novel coronavirus (2019-nCoV) in vitro. Cell Res. 2020;30 (3):269-271. https://doi.org/10.1038/s41422-020-0282-0

3. Spinner CD, Gottlieb RL, Criner GJ, et al. Effect of Remdesivir vs Standard Care on Clinical Status at 11 Days in Patients With Moderate COVID-19: A Randomized Clinical Trial. JAMA. Published online August 21, 2020. https:// doi.org/10.1001/jama.2020.16349

4. Pan H, Peto R, Henao-Restrepo A-M, et al. Repurposed Antiviral Drugs for Covid19 - Interim WHO Solidarity Trial Results. N Engl J Med. 2021;384(6):497-511. https://doi.org/10.1056/NEJMoa2023184

5. Wang Y, Zhang D, Du G, et al. Remdesivir in adults with severe COVID-19: a randomised, double-blind, placebo-controlled, multicentre trial. The Lancet. Published online 2020. https://doi.org/10.1016/S0140-6736(20)31022-9

6. Beigel JH, Tomashek KM, Dodd LE, et al. Remdesivir for the Treatment of Covid19 - Final Report. N Engl J Med. Published online October 8, 2020. https:// doi.org/10.1056/NEJMoa2007764 
7. Goldman JD, Lye DCB, Hui DS, et al. Remdesivir for 5 or 10 Days in Patients with Severe Covid-19. N Engl J Med. 2020;0(0):null. https://doi.org/10.1056/ NEJMoa2015301

8. Morris $\mathrm{AM}$, Bean $\mathrm{S}$, Bell $\mathrm{CM}$, et al. Strategies to manage tocilizumab shortages during the COVID-19 pandemic. Sci Briefs Ont COVID-19 Sci Advis Table. 2021;2 (22). https://doi.org/10.47326/ocsat.2021.02.22.1.0

9. Cevik M, Kuppalli K, Kindrachuk J, Peiris M. Virology, transmission, and pathogenesis of SARS-CoV-2. BMJ. 2020;371:m3862. https://doi.org/10.1136/ bmj.m3862

10. Jüni $P$, Odutayo $A$, Allen $U$, et al. Dexamethasone for Patients Hospitalized with COVID-19.; 2020. https://doi.org/10.47326/ocsat.2020.01.01.1.0

11. Sterne JAC, Murthy S, Diaz JV, et al. Association Between Administration of Systemic Corticosteroids and Mortality Among Critically III Patients With COVID19: A Meta-analysis. JAMA. Published online September 2, 2020. https:// doi.org/10.1001/jama.2020.17023

12. Morris AM, Stall NM, Bobos $P$, et al. Tocilizumab for hospitalized patients with COVID-19. Sci Briefs Ont COVID-19 Sci Advis Table. 2021;2(11). https:// doi.org/10.47326/ocsat.2021.02.11.1.0

13. Kokic G, Hillen HS, Tegunov D, et al. Mechanism of SARS-CoV-2 polymerase stalling by remdesivir. Nat Commun. 2021;12(1):279. https://doi.org/10.1038/ s41467-020-20542-0

14. Siemieniuk RA, Bartoszko JJ, Ge L, et al. Drug treatments for covid-19: living systematic review and network meta-analysis. BMJ. 2020;370:m2980. https:// doi.org/10.1136/bmj.m2980

15. Kaka AS, MacDonald R, Greer N, et al. Major update: remdesivir for adults with COVID-19: a living systematic review and meta-analysis for the American College of Physicians Practice Points. Ann Intern Med. Published online 2021. https://doi.org/10.7326/M20-8148

16. Thu VN, Gabriel F, Sarah C-B, et al. RCT studies on preventive measures and treatments for COVID-19. https://covid-nma.com/living_data/index.php? allcomp\#images2-8

17. COVID-19 Treatment Guidelines Panel. Coronavirus Disease 2019 (COVID-19) Treatment Guidelines. Published 2021. Accessed March 7, 2021. https:// www.covid19treatmentguidelines.nih.gov/

18. World Health Organization. Therapeutics and COVID-19. Published March 31, 2021. https://apps.who.int/iris/bitstream/handle/10665/340374/WHO-2019nCoV-therapeutics-2021.1-eng. pdf?sequence=1\&isAllowed =y 